Annals of Pure and Applied Mathematics

Vol.19, No.2, 2019, 175-181

ISSN: 2279-087X (P), 2279-0888(online)

Published on 20 April 2019

Annals of

www.researchmathsci.org

DOI: http://dx.doi.org/10.22457/apam.614v19n2a6

Pure and Applied

Mathematics

\title{
Multiplicative Neighborhood Indices
}

\author{
V.R. Kulli
}

Department of Mathematics

Gulbarga University, Gulbarga 585106, India

e-mail: vrkulli@gmail.com

Received 28 March 2019; accepted 14 April 2019

Abstract. We define the multiplicative total neighborhood index, multiplicative $F$ neighborhood index and generalized multiplicative version of these indices and determine exact formulas for line graphs of subdivision graphs of 2-D lattice, nanotube and nanotorus of $T U C_{4} \mathrm{C}_{8}[p, q]$.

Keywords: Multiplicative neighborhood indices, line graph, subdivision graph, nanostructures.

AMS Mathematics Subject Classification (2010): 05C07, 05C35, $05 C 90$

\section{Introduction}

Throughout this paper $G$ is a finite, simple, connected graph with vertex set $V(G)$ and edge set $E(G)$. The degree $d_{G}(v)$ of a vertex $v$ is the number of vertices adjacent to $v$. The line graph $L(G)$ of $G$ is the graph whose vertex set corresponds to the edges of $G$ such that two vertices of $L(G)$ are adjacent if the corresponding edges of $G$ are adjacent. The subdivision graph $S(G)$ of $G$ is the graph obtained from $G$ by replacing each of its edges by a path of length two. Let $N_{G}(v)=\{u: u v \in E(G)\}$. Let

$$
S_{G}(v)=\sum_{u \in N_{G}(v)} d_{G}(u)
$$

be the degree sum of neighbor vertices. For undefined term and notation, we refer to [1].

We need the following results.

Lemma 1. Let $G$ be a graph with $p$ vertices and $q$ edges. Then $S(G)$ has $p+q$ vertices and $2 q$ edges.

Lemma 2. Let $G$ be a graph with $p$ vertices and $q$ edges. Then $L(G)$ has $q$ vertices and $\frac{1}{2} \sum_{i=1} d_{G}\left(u_{i}\right)^{2}-q$ edges.

A molecular graph is a graph such that its vertices represent to the atoms and the edges to the bonds. Chemical Graph Theory has an important effect on the development of Chemical Sciences. A topological index is a numerical parameter mathematically derived from the graph structure. Numerous topological indices have been considered in Chemistry and have found some useful applications, especially in $Q S P R / Q S A R$ research see $[2,3]$. 


\section{V.R. Kulli}

Recently, the first neighborhood Zagreb index was introduced and studied by Basavangoud et al. [4] and Mondal et al. [5], defined as

$$
N M_{1}(G)=\sum_{u \in V(G)} S_{G}(u)^{2} .
$$
defined as

The fifth $M_{1}$ and $M_{2}$ Zagreb indices were introduced by Graovac et al. in [6],

$$
M_{1} G_{5}(G)=\sum_{u v \in E(G)}\left[S_{G}(u)+S_{G}(v)\right], \quad M_{2} G_{5}(G)=\sum_{u v \in E(G)} S_{G}(u) S_{G}(v) .
$$

The fifth multiplicative $M_{1}$ and $M_{2}$ Zagreb indices were proposed by Kulli in [7], defined as

$$
M_{1} G_{5} I I(G)=\prod_{u v \in E(G)}\left[S_{G}(u)+S_{G}(v)\right], \quad M_{2} G_{5} I I(G)=\prod_{u v \in E(G)} S_{G}(u) S_{G}(v) .
$$

Recently, the fifth arithmetic-geometric index [8], fourth multiplicative $\mathrm{ABC}$ index [9], fifth multiplicative arithmetic-geometric index [10], fifth multiplicative hyper Zagreb indices [11], multiplicative atom bond connectivity index [12] were introduced and studied.

In [7], Kulli introduced the multiplicative first neighborhood index, defined as

$$
N M_{1} I I(G)=\prod_{u \in V(G)} S_{G}(u)^{2} .
$$

We introduce the multiplicative total neighborhood index of a graph $G$, defined as

$$
T_{n} I I(G)=\prod_{u \in V(G)} S_{G}(u) .
$$

We propose the multiplicative $F_{1}$-neighborhood index of a graph and it is defined as

$$
F_{1} \operatorname{NII}(G)=\prod_{u \in V(G)} S_{G}(u)^{3} .
$$

We now continue the generalization and define the general first multiplicative neighborhood index of a graph $G$ as

$$
N M_{1}^{a} I I(G)=\prod_{u \in V(G)} S_{G}(u)^{a}
$$

where $a$ is a real number.

In this paper, we compute the multiplicative first neighborhood index, multiplicative total neighborhood index, multiplicative $F_{1}$-neighborhood index and the general first multiplicative neighborhood index of line graphs of subdivision graphs of 2$D$ lattice, nanotube and nanotorus of $T U C_{4} C_{8}[p, q]$.

\section{2-D lattice, nanotube and nanotorus of $T U C_{4} C_{8}[p, q]$}

In this section, the graphs of 2-D lattice, nanotube and nanotorus of $T U C_{4} \mathrm{C}_{8}[4,2]$ are shown in Figure 1. 
Multiplicative Neighborhood Indices

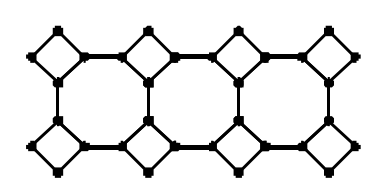

$G_{1}$

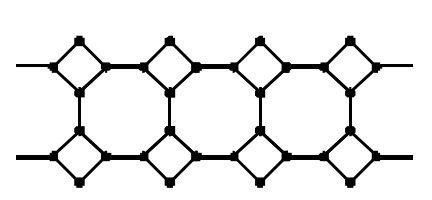

$H_{1}$

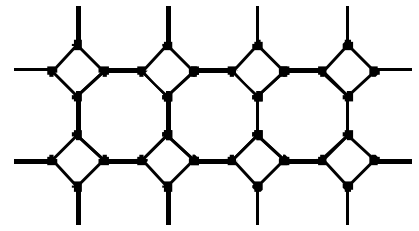

$K_{1}$

(c)

Figure 1:

(a) 2D-lattice of $T U C_{4} C_{8}[4,2]$ (b) $T U C_{4} C_{8}[4,2]$ nanotube (c) $T U C_{4} C_{8}[4,2]$ nanotorus

\section{2-D lattice of $T U C_{4} C_{8}[p, q]$}

A subdivision graph of 2-D lattice and line graph of subdivision graph of 2-D lattice of $T U C_{4} C_{8}[4,2]$ are shown in Figure 2(a) and Figure 2(b) respectively.

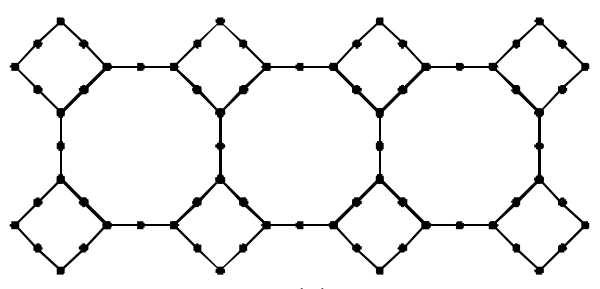

(a)

(a) subdivision graph of

2D-lattice of $\mathrm{TUC}_{4} \mathrm{C}_{8}[4,2]$

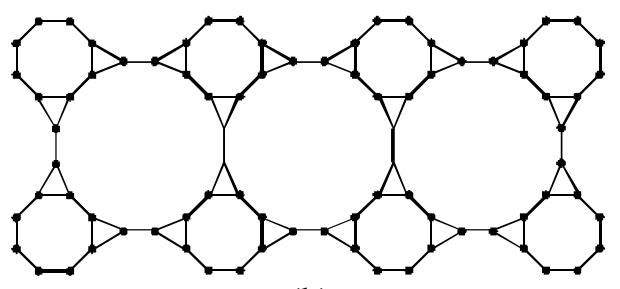

(b)

(b) line graph of the subdivision graph of 2D-lattice of $T U C_{4} C_{8}[4,2]$

Figure 2:

Let $G$ be a line graph of subdivision graph of 2-D lattice of $T U C_{4} C_{8}[p, q]$, where $p$ is the number of squares in a row and $q$ is the number of rows of squares. The graph of 2-D lattice of $T U C_{4} C_{8}[p, q]$ has $4 p q$ vertices and $6 p q-p-q$ edges. By Lemma 1, a subdivision graph of 2-D lattice of $T U C_{4} C_{8}[p, q]$ has $10 p q-p-q$ vertices and 2(6pq-p $-q)$ edges. Thus by Lemma $2, G$ has $2(6 p q-p-q)$ vertices and $18 p q-5 p-5 q$ edges. From Figure 2(b), we see that $G$ has vertices of degree 2 or 3 . The vertex partition based on the degree sum of neighbor vertices is obtained as given in Table 1 and Table 2.

\begin{tabular}{lcccc}
\hline$S_{G}(u) \backslash u \in V(G)$ & 4 & 5 & 8 & 9 \\
Number of vertices & 8 & $4(p+q-2)$ & $4(p+q-2)$ & $2(6 p q-5 p-5 q+4)$ \\
\hline
\end{tabular}

Table 1: Vertex partition of $G$ when $p>1, q>1$

\begin{tabular}{llccc}
\hline $\mathrm{S}_{\mathrm{G}}(u) \backslash u \in V(G)$ & 4 & 5 & 8 & 9 \\
Number of vertices & 8 & $4(p-1)$ & $4(p-1)$ & $2(p-1)$ \\
\hline
\end{tabular}

Table 2: Vertex partition of $G$ when $p>1, q=1$

Theorem 1. The general first multiplicative neighborhood index of a line graph of subdivision graph of 2-D lattice of $T U C_{4} C_{8}[p, q]$ is given by

$$
\begin{aligned}
N M_{1}^{a} I I(G) & =4^{8 a} \times 5^{4 a(p+q-2)} \times 8^{4 a(p+q-2)} 9^{2 a(6 p q-5 p-5 q+4)}, \text { if } p>1, q>1, \\
& =4^{8 a} \times 5^{4 a(p-1)} \times 8^{4 a(p-1)} \times 9^{2 a(p-1)}, \text { if } p>1, q=1 .
\end{aligned}
$$




\section{V.R. Kulli}

Proof: Let $G$ be a line graph of subdivision graph of 2-D lattice of $T U C_{4} C_{8}[p, q]$.

Case 1. Suppose $p>1, q>1$.

From equation (1) and by using Table 1, we deduce

$$
\begin{aligned}
N M_{1}^{a} I I(G) & =\prod_{u \in V(G)} S_{G}(u)^{a} \\
& =\left(4^{a}\right)^{8} \times\left(5^{a}\right)^{4(p+q-2)} \times\left(8^{a}\right)^{4(p+q-2)} \times\left(9^{a}\right)^{2(6 p q-5 p-5 q+4)} \\
& =4^{8 a} \times 5^{4 a(p+q-2)} \times 8^{4 a(p+q-2)} \times 9^{2 a(6 p q-5 p-5 q+4)}
\end{aligned}
$$

Case 2. Suppose $p>1, q=1$.

By using equation (1) and Table 2, we derive

$$
\begin{aligned}
N M_{1}^{a} I I(G) & =\prod_{u \in V(G)} S_{G}(u)^{a} \\
& =\left(4^{a}\right)^{8} \times\left(5^{a}\right)^{4(p-1)} \times\left(8^{a}\right)^{4(p-1)} \times\left(9^{a}\right)^{2(p-1)} \\
& =4^{8 a} \times 5^{4 a(p-1)} \times 8^{4 a(p-1)} \times 9^{2 a(p-1)} .
\end{aligned}
$$

We obtain the following results by Theorem 1 .

Corollary 1.1. The multiplicative first neighborhood index of a line graph of subdivision graph of $2-D$ lattice of $T U C_{4} C_{8}[p, q]$ is given by

$$
\begin{gathered}
N M_{1} I I(G)=4^{16} \times 5^{8(p+q-2)} \times 8^{8(p+q-2)} \times 9^{4(6 p q-5 p-5 q+4)}, \text { if } p>1, q>1, \\
=4^{16} \times 5^{8(p-1)} \times 8^{8(p-1)} \times 9^{4(p-1)}, \quad \text { if } p>1, q=1 .
\end{gathered}
$$

Proof: Put $a=2$ in equations (2) and (3), we get the desired results.

Corollary 1.2. The multiplicative total neighborhood index of a line graph of subdivision graph of 2-D lattice of $T U C_{4} C_{8}[p, q]$ is given by

$$
\begin{aligned}
T_{n} I I(G) & =4^{8} \times 5^{4(p+q-2)} \times 8^{4(p+q-2)} \times 9^{2(6 p q-5 p-5 q+4)}, & \text { if } p>1, q>1, \\
& =4^{8} \times 5^{4(p-1)} \times 8^{4(p-1)} \times 9^{2(p-1)}, & \text { if } p>1, q=1 .
\end{aligned}
$$

Proof: Put $a=1$ in equations (2) and (3), we get the desired results.

Corollary 1.3. The multiplicative $F_{1}$-neighborhood index of a line graph of subdivision graph of 2-D lattice of $T U C_{4} C_{8}[p, q]$ is given by

$$
\begin{aligned}
F_{1} N I I(G) & =4^{24} \times 5^{12(p+q-2)} \times 8^{12(p+q-2)} \times 9^{6(6 p q-5 p-5 q+4)}, & & \text { if } p>1, q>1, \\
& =4^{24} \times 5^{12(p-1)} \times 8^{12(p-1)} \times 9^{6(p-1)}, & & \text { if } p>1, q=1 .
\end{aligned}
$$

Proof: Put $a=3$ in equations (2) and (3), we obtain the desired results.

\section{4. $T U C_{4} \mathrm{C}_{8}[p, q]$ nanotubes}

In this section, a subdivision graph of $T U C_{4} C_{8}[4,2]$ nanotube and a line graph of subdivision graph of $T U C_{4} C_{8}[4,2]$ nanotube are presented in Figure 3(a) and Figure 3(b) respectively. 


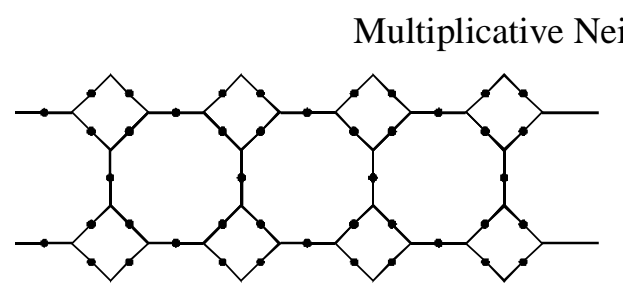

(a) Subdivision graph of $T U C_{4} C_{8}[4,2]$ nanotube

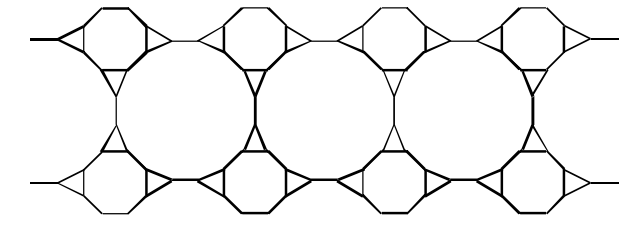

(b) Line graph of subdivision graph of $T U C_{4} C_{8}[4,2]$ nanotube

Figure 3:

Let $H$ be a line graph of subdivision graph of $T U C_{4} C_{8}[p, q]$ nanotube. A graph of $T U C_{4} C_{8}[p, q]$ nanotube has $4 p q$ vertices and $6 p q-p$ edges. By Lemma 1, a subdivision graph of $T U C_{4} C_{8}[p, q]$ nanotube has $10 p q-p$ vertices and $12 p q-2 p$ edges. Hence by Lemma $2, H$ has $12 p q-p$ vertices and $12 p q-2 p$ vertices and $18 p q-5 p$ edges. From Figure 3(b), we see that $H$ has vertices of degree 2 or 3 . The vertex partition based on the degree sum of neighbor vertices is obtained as given in Table 3 and Table 4.

\begin{tabular}{lccc}
\hline$S_{H}(u) \backslash u \in V(H)$ & 5 & 8 & 9 \\
Number of vertices & $4 p$ & $4 p$ & $12 p q-10 p$ \\
\hline \multicolumn{4}{c}{ Table 3: Vertex partition of $H$ if $p>1, q>1$} \\
\\
\hline$S_{H}(u) \backslash u \in V(H)$ & 5 & 8 & \\
Number of vertices & $4 p$ & $4 p$ & 9 \\
\hline
\end{tabular}

Table 4: Vertex partition of $H$ if $p>1, q=1$

Theorem 2. Let $H$ be a line graph of subdivision graph of $T U C_{4} C_{8}[p, q]$ nanotube. Then the general first multiplicative neighborhood index of $H$ is

$$
\begin{gathered}
N M_{1}^{a} I I(H)=5^{4 a p} \times 8^{4 a p} \times 9^{a(12 p q-10 p)}, \text { if } p>1, q>1, \\
=5^{4 a p} \times 8^{4 a p} \times 9^{2 a p}, \text { if } p>1, q=1 .
\end{gathered}
$$

Proof: Case 1. Suppose $p>1, q>1$.

By using equation (1) and Table 3 , we deduce

Case 2. Suppose $p>1, q=1$.

$$
\begin{aligned}
N M_{1}^{a} I I(H) & =\prod_{u \in V(H)} S_{H}(u)^{a} \\
& =\left(5^{a}\right)^{4 p} \times\left(8^{a}\right)^{4 p} \times\left(9^{a}\right)^{12 p q-10 p} \\
& =5^{4 a p} \times 8^{4 a p} \times 9^{a(12 p q-10 p)} .
\end{aligned}
$$

From equation (1) and by using Table 4, we derive

$$
\begin{aligned}
N M_{1}^{a} I I(H) & =\prod_{u \in V(H)} S_{H}(u)^{a} \\
& =\left(5^{a}\right)^{4 p} \times\left(8^{a}\right)^{4 p} \times\left(9^{a}\right)^{2 p} \\
& =5^{4 a p} \times 8^{4 a p} \times 9^{2 a p} .
\end{aligned}
$$

We establish the following results by Theorem 2 . 


\section{V.R. Kulli}

Corollary 2.1. The multiplicative first neighborhood index of $H$ is given by

$$
\begin{aligned}
N M_{1} I I(H) & =5^{8 p} \times 8^{8 p} \times 9^{2(12 p q-10 p)}, & & \text { if } p>1, q>1, \\
& =5^{8 p} \times 8^{8 p} \times 9^{4 p}, & & \text { if } p>1, q=1 .
\end{aligned}
$$

Proof: Put $a=2$ in equations (4) and (5), we get the desired results.

Corollary 2.2. The multiplicative total neighborhood index of $H$ is given by

$$
\begin{aligned}
T_{n} I I(H) & =5^{4 p} \times 8^{4 p} \times 9^{12 p q-10 p} & & \text { if } p>1, q>1, \\
= & 5^{4 p} \times 8^{4 p} \times 9^{2 p}, & & \text { if } p>1, q=1 .
\end{aligned}
$$

Proof: Put $a=1$ in equations (4) and (5), we get the desired results

Corollary 2.3. The multiplicative $F_{1}$-neighborhood index of $H$ is given by

$$
\begin{aligned}
F_{1} N I I(H) & =5^{12 p} \times 8^{12 p} \times 9^{3(12 p q-10 p)}, & & \text { if } p>1, q>1, \\
& =5^{12 p} \times 8^{12 p} \times 9^{6 p}, & & \text { if } p>1, q=1 .
\end{aligned}
$$

Proof: Put $a=3$ in equations (4) and (5), we obtain the desired results.

\section{5. $T U C_{4} \mathrm{C}_{8}[p, q]$ nanoturus}

In this section, a subdivision graph of $T U C_{4} C_{8}[4,2]$ nanotorus and line graph of subdivision graph of $T U C_{4} C_{8}[4,2]$ nanotorus are shown in Figure 4(a) and Figure 4(b).

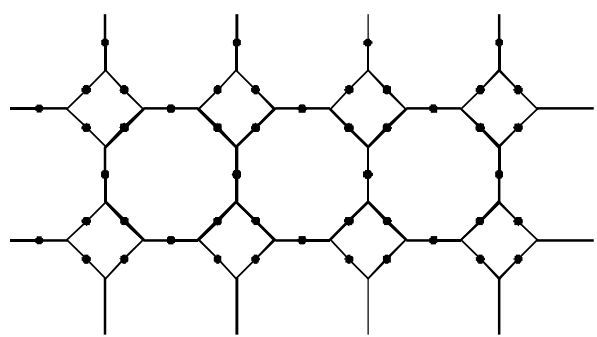

(a) Subdivision graph of $T U C_{4} C_{8}[p, q]$ nanotorus

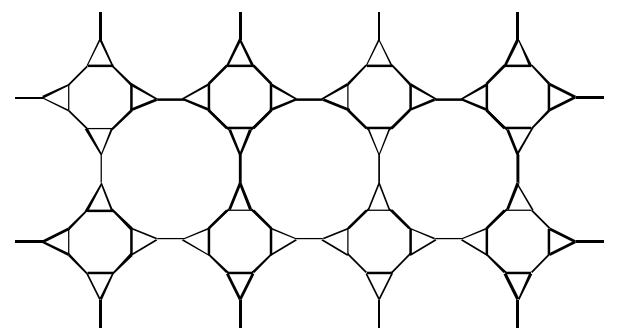

(b) Line graph of subdivision graph of $T U C_{4} C_{8}[p, q]$ nanotorus

Figure 4:

Let $K$ be a line graph of subdivision graph of $T U C_{4} C_{8}[p, q]$ nanotorus. A graph of $T U C_{4} C_{8}[p, q]$ nanotorus has $4 p q$ vertices and $6 p q$ edges. By Lemma 1, a subdivision graph of $T U C_{4} C_{8}[p, q]$ nanotorus has $10 p q$ vertices and $12 p q$ edges. Therefore by Lemma $2, K$ has $12 p q$ vertices and $18 p q$ edges. Clearly the degree of each vertex is 3 . The vertex partition based on the degree sum of neighbor vertices of each vertex is as given in Table 5.

$S_{K}(u) \backslash u \in V(K)$

9

Number of vertices

$12 p q$

Table 5: Vertex partition of $K$

Theorem 3. Let $K$ be a line graph of subdivision graph of $T U C_{4} C_{8}[p, q]$ nanotorus. Then

$$
N M_{1}^{a} I I(K)=\left(9^{a}\right)^{12 p q} \text {. }
$$

(ii) $\quad N M_{1} I I(K)=9^{24 p q}$. 
Multiplicative Neighborhood Indices

(iii) $T_{n} I I(K)=9^{12 p q}$.

(iv) $\quad F_{1} N I I(K)=9^{36 p q}$.

Proof: By using definitions and Table 5, we get the desired results.

Acknowledgement: The author is thankful to the referee for his/her suggestions.

\section{REFERENCES}

1. V.R.Kulli, College Graph Theory, Vishwa International Publications, Gulbarga, India (2012).

2. V.R.Kulli, Multiplicative Connectivity Indices of Nanostructures, LAP, Lembert Academic Publishing (2018).

3. R.Todeschini and V.Consonni, Molecular Descriptors for Chemoinformatics, WileyVCH, Weinheim, (2009).

4. B.Basavanagoud, A.P.Barangi and S.M.Hosamani, First neighbourhood Zagreb index of some nanostructures, Proceedings IAM. 7(2) (2018) 178-193.

5. S.Mondal, N.De and A.Pal, On neighborhood index of product of graphs, ArXiv : 1805.05273vi [Math. Co] 14 May 2018.

6. A.Graovac, M.Ghorbani and M.A.Hosseinzadeh, Computing fifth geometricarithmetic index of nanostar dendrimers, Journal of Mathematical Nanoscience, 1(1) (2011) 33-42.

7. V.R.Kulli, Some new multiplicative geometric-arithmetic index, Journal of Ultra Scientist of Physical Sciences, A, 29(2) (2017) 52-57.

8. V.R.Kulli, New arithmetic-geometric indices, Annals of Pure and Applied Mathematics, 13(2) (2017) 165-172.

9. V.R.Kulli, Two new multiplicative atom bond connectivity indices, Annals of Pure and Applied Mathematics, 13(1) (2017) 1-7.

10. V.R.Kulli, New multiplicative arithmetic-geometric indices, Journal of Ultra Scientist of Physical Sciences, A, 29(6) (2017) 205-211.

11. V.R.Kulli, Some new fifth multiplicative Zagreb indices of PAMAM dendrimers, Journal of Global Research in Mathematics, 5(2) (2018) 82-86.

12. V.R.Kulli and M.H.Akhabari, Multiplicative atom bond connectivity and multiplicative geometric-arithmetic indices of dendrimer nanostars, Annals of Pure and Applied Mathematics, 16(2) (2018) 429-436. 